\title{
Light chain deposition disease involving the airways: diagnosis by fibreoptic bronchoscopy
}

\author{
M. Colombat*, V. Gounant" ${ }^{\#}$ H. Mal" ${ }^{*}$ P. Callard* and B. Milleron ${ }^{\#}$
}

ABSTRACT: Light chain deposition disease (LCDD) infrequently affects the lungs and usually causes damage to the parenchyma, while bronchial involvement appears to be very rare. The present authors report the case of a 64-yr-old female with LCDD characterised by asymptomatic airway involvement.

Ten months after excision of a poorly differentiated vaginal carcinoma, a routine chest computed tomography (CT) scan revealed two lung cysts, several bilateral nodules and diffuse bronchial thickening. Pulmonary function tests were normal. Fibreoptic bronchoscopy showed marked diffuse mucosal thickening with highly conspicuous vascular plexuses. Nonamyloidal deposits were found in the bronchial wall, but no definite diagnosis could be proposed.

On follow-up, the patient was still asymptomatic and the CT scan and endoscopic appearance remained unchanged. The final diagnosis of $\kappa$ LCDD was established 18 months later by another series of bronchial biopsies with frozen samples. Interestingly, electron microscopy showed dense granular deposits associated with nonamyloidal fibrils. An increased number of lung cysts were observed $\mathbf{3 2}$ months after identification of bronchial abnormalities, confirming the progressive nature of the disease. No extrapulmonary deposits or immunoproliferative disorder were found.

In conclusion, light chain deposition disease, which may remain latent for several years, can entirely involve large airways and may be diagnosed by bronchial biopsy.

KEYWORDS: Airways, fibreoptic bronchoscopy, light chain deposition disease

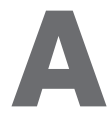

part from their role in associated haematological diseases, monoclonal immunoglobulins (Igs) may be directly pathogenic, particularly via tissue deposition, as mainly demonstrated in the kidney. These frequent deposits correspond to either the whole Ig or Ig subunits [1]. The deposits have various ultrastructural appearances: casts, crystals, fibrils, microtubules and granular features [1-3]. A granular appearance is characteristically observed in light chain deposition disease (LCDD), but not in amyloidosis (fibrils) [1-4]. The term LCDD, described in 1976 by RANDALL et al. [5], is restricted to nonamyloidal forms of light chain deposition. Microscopically, the deposits appear as amorphous material without a $\beta$-pleated sheet configuration, which consequently does not bind Congo red stain, in contrast with amyloidosis [4, 6-8]. Immunofluorescence studies reveal positivity for $\kappa$ but not for $\lambda$ light chains in contrast with Ig lightchain amyloidosis [4, 6-8]. LCDD predominantly affects middle-aged patients and has a slight male predilection. Renal involvement is an almost constant feature [6, 7]. Apart from the kidneys, the liver and heart are the organs most frequently involved [4, 6, 7]. Lung involvement is particularly uncommon, but can sometimes dominate the clinical course of LCDD and lead to severe respiratory insufficiency [9]. The exceptional possibility of bronchial involvement has been previously reported, but, to the present authors' knowledge, no precise description is available, especially regarding the endoscopic appearance [10]. The present case study reports one case of LCDD with diffuse asymptomatic airway involvement, in which the diagnosis was established by bronchial biopsy obtained by fibreoptic bronchoscopy.
AFFILIATIONS

*Service d'Anatomie Pathologique, Université Pierre et Marie Curie, and \# Service de Pneumologie, Hôpital Tenon, and

'Service de Pneumologie, Hôpital Bichat, Paris, France.

CORRESPONDENCE M. Colombat Service d'Anatomie Pathologique Hôpital Tenon 4 Rue de la Chine 75020 Paris France Fax: 33156017876 E-mail: colombatm@yahoo.fr

Received:

October 142006

Accepted after revision: December 222006

STATEMENT OF INTEREST None declared.

European Respiratory Journal Print ISSN 0903-1936 Online ISSN 1399-3003 


\section{CASE REPORT}

A 64-yr-old female was referred to the present authors' Dept of Respiratory Medicine at Tenon Hospital (Paris, France), for evaluation of lung abnormalities discovered on a routine chest computed tomography (CT) scan performed 10 months after excision of a poorly differentiated vaginal carcinoma. The patient did not complain of any symptoms and had no history of smoking. Physical examination was normal. A chest CT scan demonstrated two round cystic airspaces in the right lung (middle lobe and lower lobe) measuring $0.5-2 \mathrm{~cm}$. Several bilateral nodules measuring up to $1 \mathrm{~cm}$ and diffuse bronchial thickening were also observed (fig. 1). Hilar and mediastinal lymph nodes were not enlarged. Pulmonary function tests were normal. Fibreoptic endoscopy revealed a diffuse, inflamed and thickened mucosa reducing the bronchial lumen. The mucosal vascular plexus was also highly conspicuous (fig. 2). Microscopic examination of bronchial biopsies demonstrated abundant extracellular amorphous eosinophilic deposits in the lamina propria, vessel walls and along the basement membrane just beneath a normal respiratory epithelium. This material was partly surrounded by multinucleated giant cells. Congo red staining did not display any apple-green birefringence in polarised light. No tumour cells were observed. The conclusion of histological examination was "nonamyloidal immune deposits in the bronchial wall", and no definite diagnosis was established. Since 18-F-fluoro-2deoxyglucose positron emission tomography did not display any F-deoxyglucose uptake, no further investigations were performed. On follow-up, the clinical course was uneventful, and the patient remained asymptomatic. Similarly, the abnormalities observed on the chest CT scan did not progress. Another bronchoscopy with bronchial biopsies was performed 18 months after the initial evaluation. The macroscopic findings remained unchanged. Histological examination revealed deposits similar to those described on the previous examination (fig. 3a). Immunofluorescence on frozen tissue showed labelling of the deposits with the anti-k light chain antibody, but not with the anti- $\lambda$ light chain antibody (fig. 3b). Electron microscopy demonstrated coarsely granular, nonorganised deposits associated with nonamyloidal fibrils (fig. 3c). Therefore, a diagnosis of $\kappa$ LCDD was adopted. No monoclonal peak was detected on either serum or urine immunoelectrophoresis. Hepatic and renal functions were normal and there was no proteinuria. No abnormality was detected on echocardiography, no malignant lymphoproliferation was observed on bone marrow biopsy and no deposits were detected on salivary gland biopsy. The patient was still asymptomatic 32 months after identification of the bronchial abnormalities, but another chest CT scan showed an increased number of lung cysts, confirming the progressive nature of the disease.

\section{DISCUSSION}

The present case study illustrates the fact that LCDD: 1) may involve large airways; 2) can be diagnosed by bronchial biopsy; 3) may be composed of mixed granular and nonamyloidal fibrils; and 4) can remain latent for several years.

In contrast to the kidney, liver and heart, little is known about lung involvement in LCDD. The possibility of lung nodules and diffuse pulmonary interstitial disease has been reported,

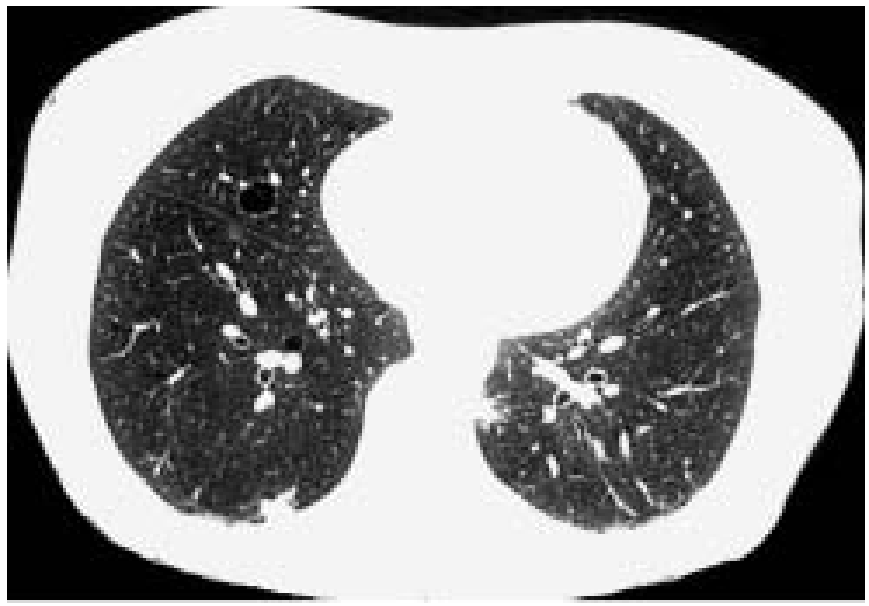

FIGURE 1. High-resolution computed tomography scan demonstrating two distinct round thin-walled cystic airspaces distributed in the right lung (middle and lower lobes), bilateral irregularly shaped nodules and bronchial thickening.

but, to date, only seven patients with pulmonary nodular-type LCDD have been reported in the literature [11-15]. These patients were mainly asymptomatic. The pulmonary nodules were multiple or unique and varied in size from $0.7-4 \mathrm{~cm}$. An underlying haematological disease was detected in four patients: lung plasmocytoma in two patients and lymphoplasmocytic lymphoma in the other two patients [11-14]. Extrapulmonary light chain deposition was not found in these seven patients. Diffuse pulmonary interstitial infiltration may be either part of a multiorgan involvement or limited to the lung. Pulmonary interstitial deposits in systemic LCDD are rarely responsible for respiratory failure unless they severely

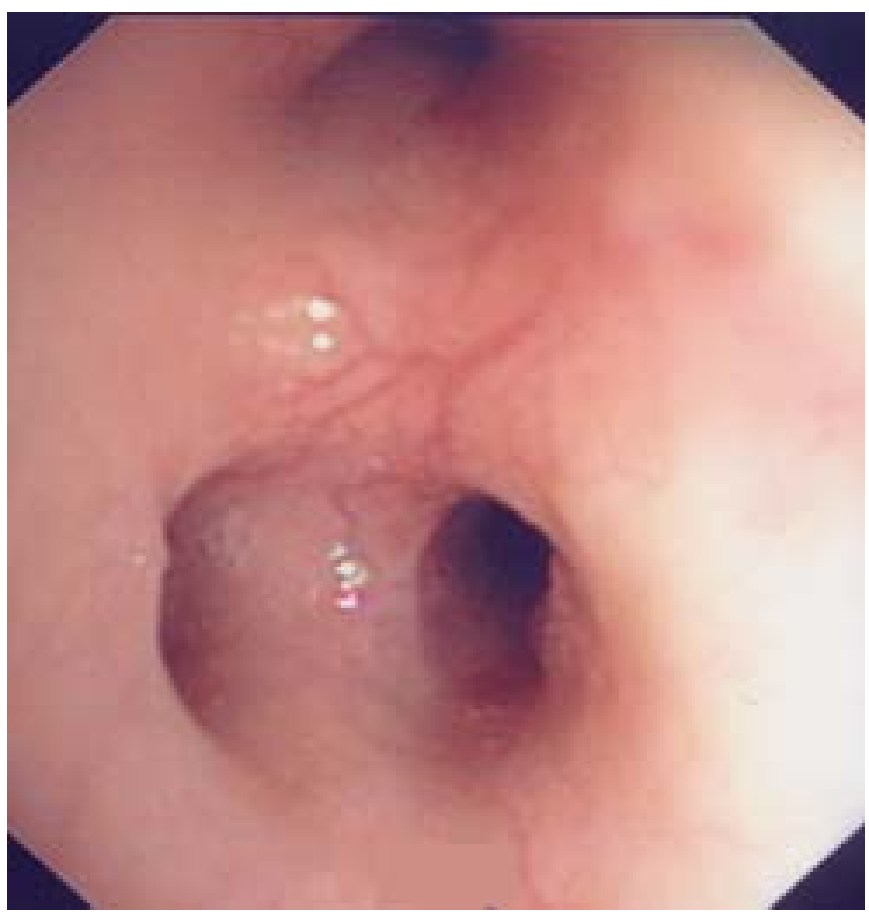

FIGURE 2. Endoscopic view of the airways. The bronchial mucosa is markedly thickened with a highly conspicuous vascular plexus. 

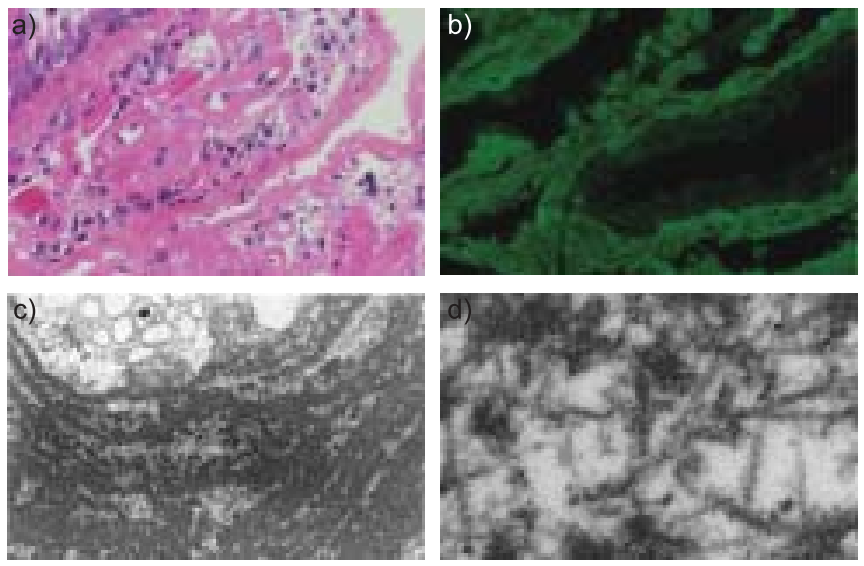

FIGURE 3. Histological, immunofluorescence and ultrastructural findings. a) Amorphous eosinophilic deposits can be seen in the vessel walls and along the basement membrane just beneath a normal respiratory epithelium. b) Direct immunofluorescence with anti-к antibody stains the vessel walls and the basement membrane of the respiratory epithelium. c) On electron microscopy, the deposits appear as granular electron-dense deposits mixed with nonamyloidal fibrils. d) An enlarged view of the granular electron-dense deposits seen in c).

affect gas exchange in the alveolar structures [16, 17]. The present authors have recently described three cases of cystic LCDD with severe chronic respiratory failure leading to lung transplantation [9]. The patients were aged 30-36 yrs at the time of onset of the symptoms. The patients presented with a progressive obstructive pulmonary pattern associated with numerous cysts distributed in both lungs. Repeated CT scans demonstrated a progressive increase in the number of lung cysts in all of these patients, and the concomitant development of pulmonary nodules in one case. Morphological analysis showed nonamyloidal deposits corresponding to $\kappa$ light chains located in the alveolar wall, vessels and small airways. No immunoproliferative disorder was identified. Slight extrapulmonary deposits were found in the salivary glands in one patient.

Very few data are available concerning the large airways in LCDD. In the three patients with cystic lung LCDD, no endoscopic abnormalities were noted and bronchial biopsies were normal (M. Colombat, unpublished data). Conversely, bronchial involvement was reported in a 34-yr-old female with multiorgan disorder revealed by recurrent respiratory infections [10]. Unfortunately, no details concerning the endobronchial macroscopic appearance were provided.

The present case study confirms that the large airways may be involved by LCDD and may be responsible for marked mucosal thickening, a particular endoscopic appearance mainly observed in a context of bronchial amyloidosis and lymphangitic carcinomatosis.

The diagnosis of LCDD is usually established by histology (renal or liver biopsy). The present case demonstrates that the diagnosis can also be obtained by bronchial biopsy. Regardless of the type of tissue sample, the diagnosis of LCDD requires microscopic and immunofluorescence analysis. Although electron microscopy is not necessary for the diagnosis, it is valuable to determine the pattern of deposition: organised, nonorganised or both. In the present patient, the ultrastructural appearance was particularly interesting as it exhibited a combination of nonorganised granular osmiophilic deposits and organised fibrillar deposits. The randomly distributed fibrils were considered to be nonamyloidal because they were thicker than amyloid fibrils. These mixed deposits are very infrequently encountered in LCDD, e.g. one out of 51 patients in the series by TOUCHARD et al. [2].

In conclusion, light chain deposition disease may affect large airways with a particular endoscopic pattern showing diffuse thickening of the mucosa. In such cases, bronchial biopsy is very helpful and analysis of a frozen sample is extremely valuable to establish the correct diagnosis of light chain deposition disease.

\section{ACKNOWLEDGEMENTS}

The authors wish to thank J.F. Bernaudin, J.C. Brouet, G. Touchard and Y. Uzunhan for their help in the preparation of this manuscript.

\section{REFERENCES}

1 Santostefano M, Zanchelli F, Zaccaria A, Poletti G, Fusaroli M. The ultrastructural basis of renal pathology in monoclonal gammopathies. J Nephrol 2005; 18: 659-675.

2 Touchard G, Bridoux F, Goujon JM. Glomérulopathies à dépots organisés d'immunoglobulines. [Glomerulonephritis with organized immunoglobulin deposits.]. Néphrologie et Thérapeutique 2005; 1: 355-364.

3 Touchard G. Ultrastructural pattern and classification of renal monoclonal immunoglobulin deposits. In: Touchard G, Hermine O, Aucouturier P, Ronco P, eds. Monoclonal Gammopathies and the Kidney. Dodrecht, Boston, London, Kluwer Academic Publishers, 2003; pp. 95-120.

4 Grogan TM, Van Camp B, Kyle RA, Müller-Hemerlink HK, Harris NL. Plasma cell neoplasm. In: Jaffe ES, Lee Harris $\mathrm{N}$, Stein $\mathrm{H}$, eds. Tumours of Haematopoietic and Lymphoid Tissues. Lyon, IARC Press, 2001; pp. 142-156.

5 Randall RE, Williamson WC, Mullinax F, Tung MY, Still WJS. Manifestations of systemic light chain deposition. Am J Med 1976; 60: 293-299.

6 Buxbaum J, Gallo G. Nonamyloidotic monoclonal immunoglobulin deposition disease. Light chain, heavy-chain, and light and heavy-chain deposition diseases. Hematol Oncol Clin North Am 1999; 13: 1235-1248.

7 Ronco PM, Alyanakian MA, Mougenot B, Aucouturier P. Light chain deposition disease: a model of glomerulosclerosis defined at the molecular level. J Am Soc Nephrol 2001; 12: 1558-1565.

8 Travis WD, Colby TV, Koss MN, Rosado-de-Christenson ML, Müller NL, King TE. Miscellaneous diseases of uncertain etiology. In: Travis WD, Colby TV, Koss MN, Rosadode-Christenson ML, Müller NL, King TE, eds. Non Neoplastic Disorders of the Lower Respiratory Tract. Washington, DC, American Registry of Pathology and the Armed Forces Institute of Pathology, 2002; pp. 857-900.

9 Colombat M, Stern M, Groussard O, et al. Pulmonary cystic disorder related to light chain deposition disease. Am J Respir Crit Care Med 2006; 173: 777-780. 
10 Knefati Y, Rossillon L, Sabatier JC, et al. Severe multi-organ involvement in Randall-type MIDD ( $\kappa$ LCDD) with mixed granular and non amyloid fibrillar $\kappa$ light chain deposits. In: Touchard G, Hermine O, Aucouturier P, Ronco P, eds. Monoclonal Gammapathies and the Kidney. Dodrecht, Boston, London, Kluwer Academic Publishers, 2003; pp. 255-256.

11 Khoor A, Myers JL, Tazelaar HD, Kurtin PJ. Amyloid-like nodules, including localized light-chain deposition. Clinicopathologic analysis of three cases. Am J Clin Pathol 2004; 121: 200-204.

12 Morinaga S, Watanabe H, Gemma A, et al. Plasmocytoma of the lung associated with nodular deposits of immunoglobulin. Am J Surg Pathol 1987; 11: 989-995.

13 Piard F, Yaziji N, Jarry O, et al. Solitary plasmocytoma of the lung with light chain extracellular deposits: a case report and review of the literature. Histopathology 1998; 32: 356-361.
14 Rostagno A, Fizzera G, Ylagan L, Kumar A, Ghiso J, Gallo G. Tumoral non-amyloidotic monoclonal immunoglobulin light chain deposits ("aggregoma"): presenting feature of B-cell dyscrasia in the three cases with immunohistochemical and biochemical analyses. $\mathrm{Br} J$ Haematol 2002; 119: 62-69.

15 Stokes MB, Jagirdar J, Burchstin O, Kornacki S, Kumar A, Gallo G. Nodular pulmonary immunoglobulin light chain deposits with coexistent amyloid and nonamyloid features in an HIV-infected patient. Mod Pathol 1997; 10: 1059-1065.

16 Linder J, Crocker BP, Vollmer RT, Shellburne J. Systemic kappa light-chain deposition. An ultrastructural and immunohistochemical study. Am J Surg Pathol 1983; 7: 85-93.

17 Kijner $\mathrm{CH}$, Yousem SA. Systemic light chain deposition disease presenting as multiple pulmonary nodules. A case report and review of the literature. Am J Surg Pathol 1988; 12: $405-413$. 